\title{
An Overview of Heterogeneous Asymmetric Catalysis
}

\author{
Zheng Wang, Kuiling Ding, and Yasuhiro Uozumi
}

\section{1}

\section{Introduction}

Driven by the ever-increasing demand for nonracemic chiral chemicals, the development of efficient methods to provide enantiomerically enriched products is of great current interest to both academia and industry [1-3]. Among the various approaches employed for this purpose, asymmetric catalysis constitutes one of the most general and appealing strategies in terms of chiral economy and environmental considerations [4-9]. Over the past few decades, intense research in this field has greatly expanded the scope of catalytic reactions that can be performed with high enantioselectivity and efficiency. Consequently, thousands of chiral ligands and their transition metal complexes have been developed for the homogeneous asymmetric catalysis of various organic transformations. Despite this remarkable success, however, only a few examples of asymmetric catalysis have been developed into industrial processes, and today most chiral chemicals are still produced from natural chiral building blocks or through the resolution of racemic mixtures. The main concern for this situation is the need for reusable chiral catalysts for industrial implementation. Due to the high cost of both the metal and the chiral ligands, systems that allow the straightforward separation of expensive chiral catalysts from reaction mixtures and efficient recycling are highly desirable. Whilst this is particularly important for large-scale productions, unfortunately it is usually very difficult to achieve for homogeneous catalytic processes. Another major drawback often associated with homogeneous catalytic processes is that of product contamination by metal leaching; this is particularly unacceptable for the production of fine chemicals and pharmaceuticals. Heterogeneous asymmetric catalysis-including the use of immobilized homogeneous asymmetric catalysts and chirally modified heterogeneous metal catalysts for enantioselective reactions-provides a good way to resolve such problems and has recently attracted a great deal of interest $[10,11]$. In this chapter we will briefly survey the field of heterogeneous asymmetric catalysis by summarizing the main features of some typical techniques at an introductory level. When relevant, we will present our 
personal comments on weighing up their strengths and limitations, though without delving into too much detail. For in-depth discussions and a comprehensive elaboration of each technique, the reader is referred to excellent recent reviews [12-16] and the ensuing chapters in this handbook, all of which have been written by scientists with expertise in these areas of research. The application of immobilized biocatalysts (including enzymes) in enantioselective organic synthesis, although representing an important field of catalytic research, is beyond the scope of this book.

The term catalyst immobilization can be defined as 'the transformation of a homogeneous catalyst into a heterogeneous one, which is able to be separated from the reaction mixture and preferably be reused for multiple times'. The main goal for the development of an immobilized chiral catalyst is to combine the positive aspects of a homogeneous catalyst (e.g. high activity, high enantioselectivity, good reproducibility) with those of a heterogeneous catalyst (e.g. ease of separation, stability, reusability). Over the past few decades, a number of strategies have been developed for this purpose. Depending on whether the modifications are made on the catalyst structure or on the reaction medium, the immobilization techniques can be categorized into two general classes, namely heterogenized enantioselective catalysts and multiphase (or monophase) catalysis in nonconventional media (Figure 1.1). The immobilized chiral catalysts can be further subdivided into several types:

- Insoluble chiral catalysts bearing stationary supports such as inorganic materials or organic crosslinked polymers, or homochiral organic-inorganic coordination polymeric catalysts without using any external support.

- Soluble chiral catalysts bearing linear polymer supports or dendritic ligands.

- Chiral catalysts with some form of nonconventional reaction medium as the 'mobile carrier', such as aqueous phase, fluorous phase, ionic liquid or supercritical carbon dioxide $\left(\mathrm{scCO}_{2}\right)$.

In the latter case, these liquids can form biphasic systems with the immiscible organic product liquid, thus giving rise to the possibility of an easy isolation and recovery of the chiral catalysts by phase separation. An important option to the

Immobilization of Asymmetric Homogeneous Catalysts

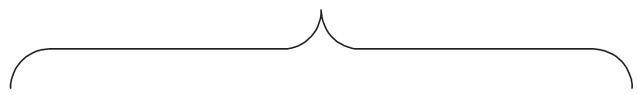

Catalyst Heterogenization

Non-Conventional Media
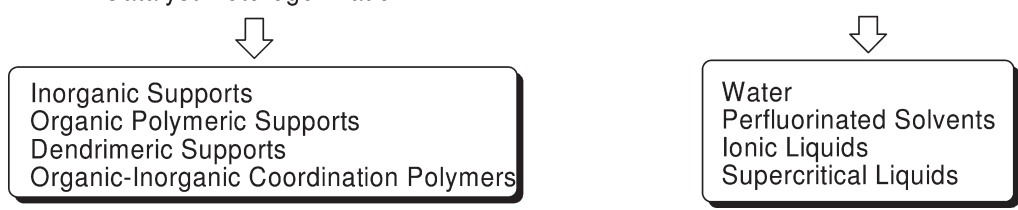

Figure 1.1 Immobilization of asymmetric homogeneous catalysts. 
biphasic catalysis is asymmetric phase-transfer catalysis (PTC), where a nonracemic chiral additive is applied to increase the mobility of a given catalyst or reactant into a favored phase and furthermore control the stereochemical outcome. Although, in general, catalyst recycling is somewhat difficult in PTC systems, it represents a distinct type of heterogeneous asymmetric catalysis.

In order to apply an immobilized chiral catalyst to a chemical process, it is often necessary to make a critical evaluation in terms of its activity, productivity, enantioselectivity, stability, ease of recovery and reusability, and so on. An ideal immobilized catalyst should not only exhibit activity and selectivity comparable or superior to its homogeneous counterpart, but also be easily recoverable from the reaction stream without metal leaching, and reusable for many runs without any loss of catalytic performance. Unfortunately, this is seldom found in real-world cases, and numerous problems can occur during the immobilization of a homogeneous catalyst. For example, in a supported chiral catalyst, one often-observed negative effect is the lower catalytic activity (even complete deactivation) compared to a homogeneous catalyst, as a result of the poor accessibility of the active sites in the solid matrix. On the other hand, the geometry of an optimized homogeneous catalyst can be unintentionally disturbed by interactions with the support, and this often leads to a negative change in enantioselectivity. For these reasons, it is a common practice to use a linker of sufficient length to connect the complex and the support, so that the complex can move far away from the solid surface and into the liquid phase.

One general requirement for the reusability of any recoverable catalyst is that both the support material and the catalytic sites must be sufficiently stable to maintain the catalytic activity during the recycling process. Any type of poor stability in the catalytic moiety or the linker part and/or incompatibility of the support with the solvent may result in leaching of the metal and/or ligand. For this reason, the support material and the linkage for immobilization should have good mechanical, thermal and chemical stabilities in order to withstand the reaction conditions used in the catalytic process. In addition, the issue of the robustness of the complex itself can be nontrivial. Immobilization is sometimes found to decrease complex degradation by virtue of steric constraints imposed by the supporting matrix, and thus may improve the stability of an immobilized catalyst relative to its homogeneous analogues. Nevertheless, this beneficial effect of immobilization on catalyst stability cannot be taken for granted, as other factors-such as the presence of strong acids or oxidizing or reducing reagents or other harsh conditions-may lead to demetallation or ligand degradation of the complex. For these reasons, the stability issue of an immobilized catalyst must be addressed on a case-by-case basis, by including the data on catalyst leaching into the product phase for assessing the potential degree of catalyst decomposition. Despite these difficulties, major efforts continue to be made to develop more efficient and practical immobilization methods for homogeneous chiral catalysts. In this regard, numerous immobilized asymmetric catalytic systems have been examined over a broad range of reactions, and a number of innovative techniques for chiral catalyst immobilization have emerged during the past two decades. In favorable cases, 
higher enantioselectivities and/or improved efficiencies were observed with a heterogeneous than with its homogeneous analogue [17-19].

\section{2}

\section{Common Techniques for Immobilization of Homogeneous Asymmetric Catalysts}

\subsection{1}

\section{Chiral Catalyst Immobilization on Inorganic Materials}

By far the most commonly used immobilization method is to support the active chiral catalyst onto or into an insoluble solid, which can be either an inorganic solid or a organic polymer. Several distinct types of strategy, featuring covalent bonds or noncovalent interactions (e.g. physisorption, electrostatic interactions, H-bonding), have been employed for linking the complex to the solid support, either onto the external surface or into the interior pores (Figure 1.2). Each of these immobilization strategies has advantages and limitations with respect to the others. Covalent bonding linkage (Figure 1.2a) is the most frequently used strategy by far, and is generally assumed to furnish the strongest binding between the complex and a support. However, it is synthetically demanding since generally some special functionalization of the ligand is required, either for grafting to a preformed support or for forming an organic polymer by copolymerization with a suitable monomer. In contrast, a major advantage of noncovalent immobilization in general is the ease of catalyst preparation, often without the need for prior functionalization of the ligand. Adsorption (Figure 1.2b) represents a very facile immobilization method, as a simple impregnation procedure can be sufficient to furnish the heterogenized catalyst. Nevertheless, catalysts immobilized via adsorption tend to be nonstable when only weak van der Waals interactions are present, and this often results in extensive catalyst leaching due to the competing interactions with solvents and/or substrates. Immobilization by electrostatic interaction (Figure 1.2c) is another common and conceptually simple technique, which is applicable to heterogenization of ionic catalytic species. Here, the solid support can be either anionic or cationic, and the catalyst is adsorbed by ion-pairing.

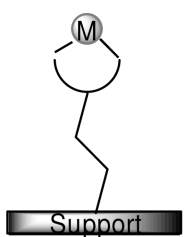

a) Covalent linkage

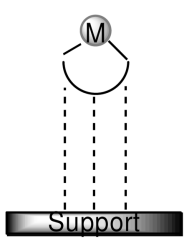

b) Adsorption

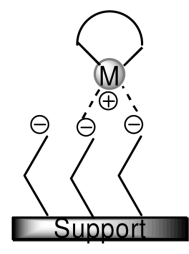

c) Electrostatic interactions

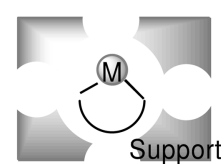

d) Entrapment

Figure 1.2 Schematic representation of the strategies for immobilizing homogeneous chiral catalysts with solid supports. 
Various supports with ion-exchange capabilities have been used for this purpose, including organic or inorganic ion-exchange resins, inorganic clays and zeolites. Although this approach can provide relatively stable immobilized catalysts, it is still limited to the catalysts which can lend themselves to immobilization through electrostatic interaction. Furthermore, competition with ionic species (either present in or produced during the reaction) in solution may result in catalyst instability and leaching. Finally, the catalytic complex can also be entrapped within the pores of some solid matrix (Figure 1.2d). In this entrapment methodology, the size of the metal complex relative to that of the window or tunnel of a porous solid is the factor of paramount importance, leading to a mechanically immobilized catalyst. This can be accomplished by preparing the complex in well-defined cages of a porous support, or alternatively by building up a polymer network around a preformed catalyst. Although conceptually very elegant, the entrapment strategy is relatively complex to implement compared with the other methods, and the size of the substrate molecules may cause diffusion problems in the catalysis. In summary, it is difficult to predict whether a covalent or a noncovalent immobilization would be preferential for a given catalyst. Although covalent bonding remains the most popular approach to chiral catalyst immobilization (mainly due to stability advantages), examples illustrated in the following chapters in this Handbook have shown that noncovalent immobilizations are gaining increasing recognition as a feasible way to achieve good stability and reusability as well as high selectivity and activity of an immobilized chiral catalyst.

One major drawback of the insoluble solid-supported chiral catalysts is that, in many cases, lower reactivities and/or poorer enantioselectivities were obtained as compared with the corresponding homogeneous catalysts. However, recent results have shown that the reverse can also be true by correctly choosing the support/ complex combination, even though this was largely achieved by trial-and-error rather than by rational design. A salient feature of the insoluble solid-supported catalysts is their easy recovery. In a batch operation, the solid-supported catalyst can be isolated from the reaction mixture by simple filtration, and in some cases can be reused for subsequent reaction cycles until deactivated. Alternatively, the heterogeneous catalysts can be employed in a continuous-flow reactor, with the advantages of easy automation and little or no reaction work-up [20-23].

Inorganic solids such as silicas, mesoporous solids (e.g. MCM-41, SBA-15), zeolites and clays have been widely used as supports for the immobilization of various homogeneous chiral catalysts [14, 24-30]. Depending on the properties of the complex and the structure of the support, the immobilization strategies can encompass the whole spectra of aforementioned interactions, from physical entrapment to covalent bonding. For example, zeolites are crystalline microporous aluminosilicates with interior cavities accessible to small reactants from the solution. A chiral metal complex with a suitable size can be assembled inside the zeolite cavity and entrapped snugly there for catalysis, as escape by diffusion through the small windows is very difficult. One advantage of the zeolite-entrapped catalyst is that the zeolite can impose shape selectivity to the catalytic system; that is, only those substrates with appropriate size and shape can reach the catalyst and 
react. Zeolite entrapment may also lead to a better catalyst stability as a result of protection of the inert framework. However, zeolite entrapment of a homogeneous chiral catalyst often leads to a decrease in enantioselectivity, presumably by a negative steric influence of the cage walls. Alternative inorganic solids were also examined as supports, for example, to immobilize the catalytic complex on the external surface of an nonporous solid such as amorphous silica, or in the interior of porous solids with void dimensions larger than zeolites. The immobilization of an electrically charged homogeneous complex by electrostatic interaction with the surface of the inorganic support is an attractive method owing to the experimental simplicity of the procedure. In this regard, lamellar solids bearing charged layers (clays such as montmorillonite K10, laponite or bentonite) have been used as supports to immobilize a variety of charged chiral metal complexes via simple anionexchange procedures.

Usually, these immobilization approaches do not necessarily require special functionalization of the ligand part. In contrast, immobilization by covalent binding of a catalyst to an inorganic support is generally accomplished by reacting complementary functional groups-one located on the solid and the other at the complex moiety-to create a new covalent bond connecting the solid and the complex. Although a large variety of functional groups have been shown to be applicable for this purpose, and despite strong immobilization (not necessarily) being expected, the approach suffered from the major drawback of a need for extensive organic synthesis. Therefore, immobilization by covalent binding would be preferential only if the stability and reusability of the resulting catalyst were to be significantly improved relative to other methods.

The use of inorganic solids can demonstrate certain advantages over other types of support. In general, the rigid framework can prevent the aggregation of active catalysts which sometimes leads to the formation of inactive multinuclear species. The chemical and thermal stabilities of the inorganic supports are also superior, rendering them compatible with a broad range of reagents and relatively harsh reaction conditions. Compared with organic polymeric supports, inorganic solids are generally superior in their mechanical properties, which makes them less prone to attrition caused by stirring and solvent attack. One negative aspect of an inorganic solid-immobilized chiral catalyst is the extreme difficulty in the characterization of the catalytic species, apart from common problems suffered by heterogenized catalysts.

The use of an inorganic support for the immobilization of homogeneous chiral catalysts has been a steadily expanding area of research during recent years, as evidenced by the numerous examples described in Chapter 2. In some cases, the heterogenization of a chiral catalyst onto an inorganic material has not only provided a facile vehicle for catalyst recycling, but also has significantly improved the catalytic performance in terms of activity, stability and/or enantioselectivity by virtue of the site-isolation and confinement effect. Taken together, it is expected that this immobilization technique will continue to play an important role in the development of highly efficient heterogeneous chiral catalysts in the future. 


\section{2 .2}

\section{Chiral Catalyst Immobilization Using Organic Polymers}

The use of organic polymer supports (either soluble or insoluble) for (chiral) catalyst immobilization is an area of considerable research interest, and is also the subject of many excellent reviews (see also Chapter 3) [31-43]. To date, a wide variety of polymer-supported chiral complexes have been prepared and tested over a broad spectrum of synthetic reactions. Some systems have demonstrated catalytic performances (activity and selectivity) rivaling their homogeneous counterparts in certain model reactions, with the additional advantages of easy recovery and reusability. By virtue of its good chemical inertness, ready availability and ease of functionalization, polystyrene crosslinked with various amounts of $p$-divinylbenzene (DVB) is the most popular insoluble organic polymer support used in chiral catalyst immobilization. For the covalent immobilization of a homogeneous catalyst to an insoluble polymer support, two different approaches have been employed, depending on whether the polymer is formed in or before the process. One approach is to use a solid-phase synthesis, starting with a preformed functionalized polymer and anchoring the suitably derivatized chiral ligand or complex by stepwise assembly of the components. A wide array of commercially available functionalized polymers with a large variety of functional groups including chloromethyl, hydroxyl, amino, thiol or pyridine rings can be used for this purpose, and a large number of chiral ligands have been immobilized to the polystyrene support using this method. Alternatively, the insoluble polymer bearing the active complex can also be prepared by copolymerization of styryl derivatives of the chiral ligand or complex with styrene and divinylbenzene.

Compared to an inorganic support, the usually low surface area of an insoluble organic polymer may limit the interfacial contact between the supported complex and the substrate. Some polymer particles can be mechanically fragile or brittle, which constitutes some limitations encountered in the recycling of polymersupported catalysts. It is generally accepted that the conformational influence, steric and polarity factors of the polymer backbones can provide a unique microenvironment for the reactants. The polymer supports have sometimes been found to exhibit beneficial impact on the catalytic reactions, leading to an enhanced selectivity and/or improved stability of the catalyst. However, in most cases, the exact role of the polymeric backbone (e.g. the linker or the degree of crosslinking) in the catalytic behavior of an immobilized metal complex is not clear and remained to be clarified.

Despite the well-known advantages of insoluble supports, there are several drawbacks in using these solids as supports for chiral catalysts due to the heterogeneous nature of the reaction conditions. The catalyst resides in the solid phase while reactants are in solution, which can often result in a decreased reaction rate owing to diffusion problems. Furthermore, the matrix effect of the solid support, though sometimes favorable, is difficult to predict and can often lead to lower enantioselectivities for the immobilized chiral catalysts than those for their 
homogeneous counterparts. Last, but not least, most of the insoluble catalysts developed so far still lack a full characterization, as the inherent heterogeneity of solid-phase systems precludes the use of many traditional analytical techniques. For these reasons, in recent years the use of soluble polymer-supported reagents and catalysts has gained significant attention as an alternative method to traditional insoluble-bound catalysts [44-52]. Soluble polymer-supported catalysts often have advantages of more facile characterization, allowing for (at least in principle) catalyst characterization on soluble polymer supports by routine analytical methods. Furthermore, the reactivity and selectivity of the soluble polymer-supported catalysts are somewhat (though not necessarily) predictable by virtue of the homogeneous nature of the solution-phase chemistry, and can be as high as those of their low-molecular-weight counterparts. Poly(ethylene glycol) (PEG) and noncrosslinked polystyrene are the most often-used polymer carriers in the preparation of soluble polymer-supported chiral catalysts. Although, anchoring of the chiral ligand/complex may be made directly to the polymer support with suitable functional groups, a linking group is often employed to impart anchor stability throughout synthesis and/or to improve accessibility to reagents. The immobilization is usually accomplished via coupling chemistry of the functionalized chiral ligands/complexes and the support. An interesting variation is that of chiral rigidrod polymers containing binaphthyl groups as the catalyst supports, where the chiral groups are an integral part of the polymer's main chain; these have also been used as soluble and recoverable polymer-bound chiral ligands/catalysts [53, 54].

One potential problem with the use of soluble polymers as recoverable catalyst supports is how to isolate the polymer from the reaction mixture. This is usually accomplished by using the various macromolecular properties of the support. Most frequently, the homogeneous solution after the reaction is simply diluted with a poor solvent (i.e. it has a poor solvating power for the polymer support), and this can induce precipitation of the polymer catalyst. The resulting heterogeneous mixture is filtered to isolate the polymer-supported catalyst, which can be recharged with the reactants and solvent for subsequent cycles of reactions. A variety of separation techniques have been employed to recover a soluble polymerbound catalyst or ligand, and the reader is referred to an elegant review on this topic [48].

\section{2 .3}

\section{Dendrimer-Supported Chiral Catalysts}

Dendrimers are a class of macromolecules with highly branched and well-defined structures, and have recently attracted much attention as soluble supports for (chiral) catalyst immobilization [55-65]. As stated above, the catalysts anchored onto or into insoluble supports often possess an uneven catalytic site distribution and partly unknown structures, and generally suffer from diminished activity due to the mass transfer limitations. Dendrimers, on the other hand, allow for the precise construction of catalyst structures with uniformly distributed catalytic 
sites - a feature which is very valuable when performing an analysis of catalytic events and mechanistic studies. It is also possible to regulate the numbers and locations of the catalytic centers at the core or at the periphery, and to fine-tune the size, shape and solubility of dendrimers by ligand design, both of which are crucial for the catalytic performance of the system. Moreover, as soluble support dendrimers can, in principle, lead to catalytic systems with activities and selectivities similar to their monomeric analogues. Since the seminal publication by Brunner in 1994-1995 on the use of chiral dendritic catalysts in asymmetric catalysis [66, 67], research in this field has achieved remarkable progress. Indeed, excellent reviews are currently available with well-documented examples demonstrating the precise definition of catalytic sites and the possibility of recovering the dendritic catalysts $[13,68]$. A variety of enantioselective dendritic catalysts are now known which, according to dendritic topology and/or the location/distribution of the catalytic sites, can be categorized into several general types, as summarized in Figure 1.3. Most of these dendritic catalysts are related to homogeneous catalysis ( $\mathrm{a}, \mathrm{b}, \mathrm{c}$ and d), but some heterogeneously supported dendritic catalysts have also been developed (e). Typically, the dendrimer can adopt the shape of either a sphere ( $\mathrm{a}$ and $\mathrm{b}$ ) or a wedge (c and d), and the chiral catalytic site(s) can be located either at the periphery (a, c and e), at the core (b) or at the focal point (d) of the

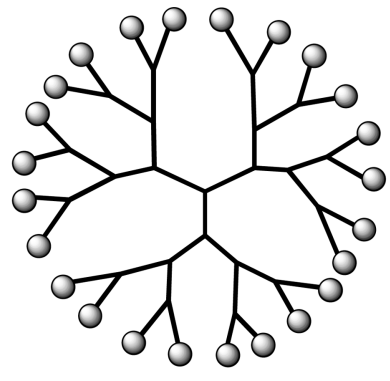

(a)

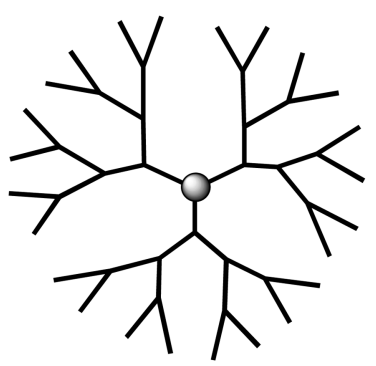

(b)

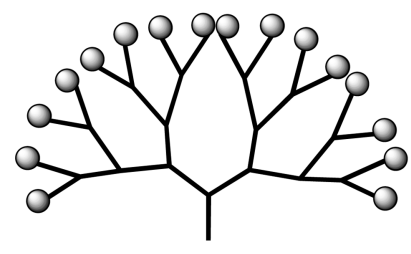

(c)

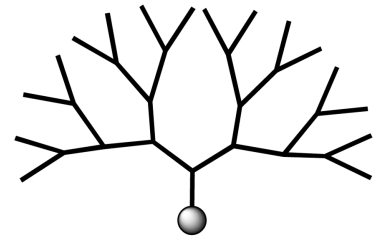

(d)

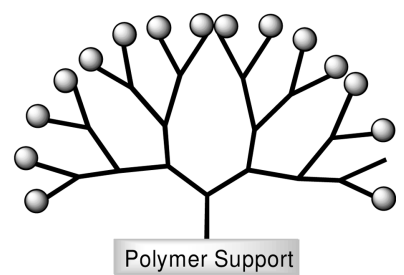

(e)

= catalytic species

Figure 1.3 Various types of dendritic catalyst with catalytic species located at the periphery (a), core (b), periphery of a wedge (c), focal point of a wedge (d), and polymer-supported dendrimer with catalytic species at the periphery (e). 
dendrimer. These diversified dendritic structures are very important for defining the catalytic performance, including the activity, enantioselectivity, stability and reusability of the catalyst system (dendritic effects). For example, the catalytic sites located at the surface of a periphery-functionalized dendrimer ( $\mathrm{a}, \mathrm{c}$ and e) would be easily accessible to the substrate, allowing reaction rates comparable to those of the homogeneous system. In addition, the multiple reaction sites in a peripheryfunctionalized dendrimer would result in high local catalyst concentrations, which can exhibit positive dendritic effects in cases where cooperative interactions between active sites are needed in the catalysis. On the other hand, for reactions that are deactivated by excess ligand, or in cases where a bimetallic deactivation mechanism is operative, core- or focal point-functionalized dendritic catalysts (b, d) would be especially beneficial owing to the site-isolation effect by the dendritic structure. The dendritic structure can also demonstrate a significant effect (negative or positive) on the enantioselectivity of an asymmetric catalysis. An elegant review of the many aspects involved in enantioselective catalysis using dendrimer supports is provided in Chapter 4 of this Handbook.

The dendritic catalysts can be recovered and reused using similar techniques as applied for other soluble polymeric catalysts discussed above, including solvent precipitation, column chromatography, two-phase catalysis or anchoring on insoluble supports. Moreover, the globular shapes and large size of the higher generations of dendrimers are well suited to catalyst-product separation by nanofiltration using a membrane, this being performed either batchwise or in a continuous-flow membrane reactor (CFMR).

It is clear that the attachment of chiral catalysts to dendrimer supports offers a potential combination of the advantages of homogeneous and heterogeneous asymmetric catalysis, and provides a very promising solution to the catalyst-product separation problem. However, one major problem which limits the practical application of these complicated macromolecules is their tedious synthesis. Thus, the development of more efficient ways to access enantioselective dendritic catalysts with high activity and reusability remains a major challenge in the near future.

\subsection{4}

\section{Self-Supported Chiral Catalysts in Asymmetric Reactions}

In the above-mentioned immobilization approaches, the chiral ligand is anchored either covalently or noncovalently to an insoluble or soluble support. Recently, another immobilization technique without the need for any external support was developed based on the assembly of multitopic chiral ligand and metals into homochiral metal-organic solids by coordination bonds [69-74]. The details of the story are described in Chapter 9 of this Handbook. In the simplest case, a ditopic or polytopic chiral ligand bearing two or more ligating units was used to link adjacent metal centers. Alternatively, chiral polyfunctional ligands were used as linkers and metal ions or clusters as nodes to construct homochiral metal-organic solids; however, the active sites were located as pendant auxiliaries 
on the main chain of the solids. In the former approach, the metal centers were incorporated into the main backbone of the resulting homochiral metal-organic assembly, where they played a dual role of structural binder and catalytically active site. Thus, it is essential that the metals used here should be capable of simultaneously bonding with at least two ligand moieties (same or different), but still have vacant or labile sites available for substrate and/or reagent coordination and activation. For the second approach, a prerequisite for the design is the orthogonal nature of the two types of functional group in the chiral bridging ligands. While the primary functional groups are responsible for connecting the network-forming metals to form extended structures, the secondary chiral groups are used to generate asymmetric catalytic sites with (or without) catalytic metals.

The modular nature of these solids allows for the fine-tuning of their catalytic performance through chemical modifications on the molecular building units. Once the chiral multitopic ligands are at hand, the synthetic protocols to assembly the homochiral solid catalysts can be quite simple. Combination of the ligand with an appropriate metal precursor in a compatible solvent would result in the spontaneous formation of polymeric structures (or oligomers). This polymerization process involves the successive formation of bonds between a multitopic ligand and a metal, and thus is facilitated by employing a ligand that shows high affinity towards the network metal. By virtue of the usually poor solubility of the solids in common organic solvents, as well as the chiral environment provided by the ligands, these self-supported chiral catalysts have demonstrated high enantioselectivities and efficiencies in a variety of heterogeneous catalytic asymmetric reactions. On completion of the reaction, the solid catalyst may be easily recovered by simple filtration and reused for several runs, without (in some reported cases) any significant loss of activity or enantioselectivity. In contrast to state-of-the-art heterogeneous catalysts which are mostly amorphous in nature, some homochiral metal-organic catalysts were even obtained in single crystal form with uniformly distributed catalytic sites, a salient feature which is highly desirable for a mechanistic understanding and structure-activity relationship (SAR) study of catalytic events which have occurred in or on a solid. The remarkable successes using self-supported metal-organic assemblies in heterogeneous (asymmetric) catalysis suggest a broader scope of application for these materials in the future.

Many challenges remain in this emerging field of heterogeneous asymmetric catalysis, however. Among other things, these solids typically maintain their structural integrity by metal-ligand coordination bonds, which encompass a broad spectrum of strengths. Therefore, beyond activity and selectivity, the stability and compatibility of the metal-organic catalysts with reaction systems is difficult to predict and should be addressed with care. To date, very few successful examples using homochiral metal-organic catalysts have been reported, and consequently further exploration of new types of homochiral assembly with a more critical assessment of their strengths and limitations in synthetic applications would be desirable. 


\section{2 .5}

\section{Chiral Catalyst Immobilization Using Nonconventional Media}

Changing the reaction medium from conventional volatile organic solvents to a form of nonconventional medium, with or without chemical manipulation on the catalyst, represents another type of general strategy used to recover and reuse a (chiral) catalytic system (see Figure 1.1) [75-83]. In general, an environmentally benign solvent is used as the nonconventional medium, as this can combine the advantages of catalyst recovery with those of 'green chemistry'. Most frequently, water, perfluorinated liquids, ionic liquids and supercritical fluids (usually supercritical $\mathrm{CO}_{2}$ ) were used as the 'green' supplants for those environmentally hazardous organic solvents. Catalysis in such a medium can be carried out under either monophasic or biphasic conditions (e.g. aqueous/organic, fluorous/organic, or other immiscible multiphasic solvent combinations), with a variable degree of solvent effects (positive or negative) on the catalytic reactivity and/or selectivity. With regards to the catalyst recovery/reuse an idealized situation is that, upon completion of the reaction, the products (in organic phase) are effectively isolated by decantation, extraction, distillation or other routine phase-separation technique, while the catalyst remains in the reaction medium ready for reuse and without any decay in activity or selectivity. The potential advantages of these systems include easy catalyst separation and recovery, a reduced environmental impact of common organic solvents and increased safety-all of which are important concerns in the development of industrial catalytic asymmetric processes.

\subsubsection{Catalyst Immobilization in Water}

Water is abundant, cheap, nonflammable, nontoxic and safe to the environment, and is the solvent used by Nature for many biological organic functional group transformations catalyzed by enzymes. For these reasons, water as a reaction medium has attracted much interest in recent years. Indeed, a diverse range of synthetic transformations can be performed in water, and completely new reactivities have been identified in some previously inaccessible reactions. Several excellent reviews are available on this topic [84-89]. One additional advantage of water important for catalysis is that it allows for easy catalyst separation and is recycled via a biphasic catalysis, by virtue of its low miscibility with most organic compounds [90-93]. One classical aqueous/organic biphasic catalytic system which has been successfully commercialized is in the hydroformylation of propene using a sodium salt of sulfonated triphenylphosphine as ligand; this renders the rhodiumbased catalyst soluble in water and facilitates its separation from the organic phase containing the product, butanal [94]. Given these facts, it is not surprising to see that asymmetric catalysis with a (recoverable) catalyst in water should represent one of the most attractive goals for synthetic chemists [95-99].

However, it is often more difficult to achieve high catalytic performance in water than in organic media, partly as a result of the generally poor water-compatibility of both the catalysts and/or the organic substrates. Several approaches have been invoked to achieve asymmetric catalysis in water, including hydrophilic modifica- 
tion of the chiral catalysts by sulfonation or quaternary cation salt formation, the use of chiral amphiphilic additives such as surfactants or phase-transfer catalysts, natural or synthetic water-soluble polymer-supported chiral catalysts, and so on. An insightful discussion on an alternative approach is provided in Chapter 6 of this Handbook, where insoluble polymer-supported chiral catalysts (chiral palladium phosphine complexes supported on TentaGel-type amphiphilic polymer bearing PEG chains) are used for heterogeneous asymmetric processes in water. As illustrated by the elegant examples, this approach combines the advantages of both aqueous reaction and ease of catalyst recovery in one system, with activity and selectivity rivaling that of their homogeneous counterparts. It should be noted that, despite the significant progresses made so far, the potential of polymer-supported chiral catalysts for aqueous reactions has not yet been fully exploited and the field is still far from mature. Hence, it remains an important issue-and a great challenge-to develop recoverable chiral catalyst systems in water, and the number of such systems will surely increase steadily in the future.

\subsubsection{Fluorous Phase-Separation Techniques in Catalysis}

Fluorous fluids such as perfluoroalkanes and perfluorodialkyl ethers are chemically inert and usually immiscible with most common organic solvents at ambient temperature. Their miscibility with organic solvents, however, improves remarkably with an increase in temperature. This special (thermotropic) solubility feature makes one-phase reaction and two-phase separation possible with a fluorous/ organic solvent mixture. Pioneered by the seminal studies of Horváth and Rábai in 1994 for the use of fluorous biphase systems in rhodium-catalyzed hydroformylation [100], and which resulted in facile catalyst separation and reuse as well as high productivities, fluorous biphasic catalysis has attracted much attention as a powerful tool to facilitate catalyst/product separation [101-109]. In these systems, the reagent, substrate and product are dissolved in an organic solvent, which is in contact with a fluorous phase containing a fluorous-soluble catalyst. Analogous in principle to aqueous/organic biphasic catalyses based on water-soluble catalysts, the commonly used hydrocarbon-soluble catalysts must be modified to a 'fluorouslike' form in order to dissolve preferentially in the fluorous phase. This is usually accomplished by attaching perfluoralkyl chains ('fluorous ponytails') of appropriate size, shape and number to the ligand core of the catalyst, which would render the molecular catalyst fluorous-soluble. In order to diminish the potentially undesirable electron-withdrawing effects of the fluorous ponytails, it is often necessary to insert some spacer groups (such as $-\mathrm{CH}_{2} \mathrm{CH}_{2} \mathrm{CH}_{2}-$ ) between the fluorous ponytail and the ligand backbone. The reaction involving the perfluoro-tagged catalyst in a fluorous and organic solvent mixture could proceed either heterogeneously with the catalyst in the fluorous phase and the substrate/reagents in the organic phase, or homogeneously since some fluorous biphasic systems can become single-phase at elevated reaction temperatures. On completion of the reaction, the mixture is cooled to room temperature and this results in a restoration of the two immiscible phases. The phases are then easily separated, with the product in the organic phase and the perfluoro-tagged catalyst in the fluorous 
layer; the catalyst can subsequently be reused for further runs in some cases. While fluorous biphase catalysis has received considerable interest since 1994, and has been the subject of several recent reviews, asymmetric fluorous catalysis has only been developed during the past decade as a novel technique that allows easy separation and recycling of the expensive chiral catalysts [110]. An overview of enantioselective fluorous catalysis, including both fluorous biphase catalysis and homogeneous enantioselective reactions promoted by fluorous catalysts is provided in Chapter 5. The chapter also outlines the remarkable achievements and research directions in this rapidly developing field.

\subsubsection{Catalytic Reactions in lonic Liquids}

The use of ionic liquids as novel reaction media has generated considerable interest over the past decade, both as a convenient solution to environmental contamination problems caused by volatile organic solvents and as an efficient method for recycling homogeneous catalysts [111-123]. Ionic liquids are salts with a low melting point $\left(<100^{\circ} \mathrm{C}\right)$ and a negligible vapor pressure; they produce no atmospheric pollution and thus are regarded as 'green' solvents. Structurally, ionic liquids are generally composed of organic cations including alkylammonium, alkylphosphonium, $N, N^{\prime}$-dialkylimidazolium and $N$-alkyl pyridinium cations, and weakly coordinating inorganic anions such as $\mathrm{PF}_{6}^{-}$or $\mathrm{BF}_{4}^{-}, \mathrm{SbF}_{6}^{-}$, and so on. Some ionic liquids are commercially available, or can be prepared via direct quaternization of the appropriate amine or phosphane followed by subsequent anion exchange. It should be noted that the physical or chemical properties of an ionic liquid (e.g. melting behavior, polarity, hydrophilicity/lipophilicity, acidity, stability, etc.) are profoundly affected by the nature of its structural constituents, and as a result the properties can in principle be modulated and fine-tuned to the reaction by the suitable combination of cation and anion. For example, variation of the chain lengths of the alkyl groups on the cation has an effect on the lipophilicity of the ionic liquid, thus allowing its handling properties (miscibility with water or organic solvents) to be adjusted.

Ionic liquids have been used as solvents for a large number of catalytic reactions [111-123]. Ionic liquids represent a class of highly polar solvents; they can dissolve a wide range of organic, inorganic and organometallic compounds, and also tend to be immiscible with nonpolar solvents (i.e. hydrocarbons, diethyl ether). Ionic liquids have an especially high affinity for ionic or polar transition-metal complexes, which allows for the immobilization of many ionic catalysts within them, without modification of the ligands. The catalytic reactions in ionic liquids can be conducted either in a single phase by dissolving in them both substrate and catalyst, or in a biphasic system whereby the catalyst resides in the ionic liquid and the substrate/product in the second phase. Upon completion of the reaction, the product can be isolated by decantation, distillation or extraction with an organic solvent (or supercritical $\mathrm{CO}_{2}$ ). In any cases, the catalyst should remain dissolved in the ionic liquid during the separation, and both the catalyst and the ionic liquid can be recovered and reused for subsequent runs. 
Although the first example of homogeneous transition-metal catalysis in an ionic liquid was reported by Parshall in 1972 [124], the subject was largely neglected at the time; however, research into this area was revived about two decades later. The use of ionic liquids in asymmetric catalysis was reported even later, beginning with Chauvin's report on a catalytic asymmetric hydrogenation and hydroformylation of alkenes in 1995 [125]. Since then, enantioselective catalysis in ionic liquids has attracted remarkable interest as an approach to the facile recycling of expensive chiral ligands and catalysts, and a range of enantioselective catalytic transformations have been examined in ionic liquids [126]. In many cases, ionic liquids have a beneficial effect on the activities and enantioselectivities, and demonstrate facile recovery and reusability of the ionic solvent-catalyst systems. The reader is referred to Chapter 7 for an excellent review on the development of enantioselective catalysis in ionic liquids.

\subsubsection{Enantioselective Catalysis in Supercritical Carbon Dioxide}

Over the past decade, the use of supercritical fluids (SCF) as reaction media has begun to attract increasing attention in synthetic organic chemistry [127-134]. Both, homogeneously and heterogeneously catalyzed reactions have been performed in SCFs as reaction media, and for details on the properties and catalytic applications of SCFs the reader is referred to a thematic issue in Chemical Reviews [127]. Generally speaking, a SCF is a fluid at a state (conditions) above its critical point (the critical temperature $T_{\mathrm{c}}$ and pressure $P_{\mathrm{c}}$ ), where upon it demonstrates both liquid-like (capable of dissolving many organic compounds) and gas-like (flow like gases and total miscibility with gaseous reactants such as $\mathrm{H}_{2}, \mathrm{CO}, \mathrm{O}_{2}$, etc.) properties, but with no gas-liquid boundaries. The solvent properties of SCFs, such as dielectric constants, viscosity and solubility, can be easily fine-tuned by adjusting the temperature and pressure or addition of cosolvents, and this provides an attractive method for controlling the rates and selectivities of catalytic reactions. The excellent miscibility of SCFs with gases tends to make them beneficial for reactions involving gases, such as hydrogenation, hydroformylation and oxidation with oxygen, where the solubility of the gaseous reactant can be rate-limiting. Among the various SCFs that have been used as reaction media to date, supercritical carbon dioxide $\left(\mathrm{scCO}_{2}\right)$ is especially attractive in that it is nontoxic, nonflammable, chemically inert to a wide range of reaction conditions, and becomes supercritical at mild conditions $\left(T_{\mathrm{c}}=31.1^{\circ} \mathrm{C}\right.$ and $P_{\mathrm{c}}=73.8$ bar $)$. Today, $\mathrm{scCO}_{2}$ is widely recognized as an environmentally benign solvent, and much attention has been focused on its use in various applications as an alternative to the environmentally damaging organic solvents. Since the first report of asymmetric hydrogenation reactions in $\mathrm{scCO}_{2}$ in 1995 [135], some progress has been made in the catalytic asymmetric reactions in SCFs (predominantly $\mathrm{ScCO}_{2}$ ), as summarized in an excellent recent review [136]. Several types of catalytic asymmetric reaction (mainly hydrogenation and hydroformylation) have been investigated in $\mathrm{scCO}_{2}$, with excellent results being obtained in some cases. The use of perfluoro tags on the ligands can considerably increase the otherwise often poor solubility of a polar 
chiral catalyst in the nonpolar $\mathrm{ScCO}_{2}$, allowing the reaction to be carried out in a single supercritical phase. A degree of success has also been achieved in the development of new protocols that allow for the separation and reuse of the catalyst in $\mathrm{scCO}_{2}$ media, by use of the so-called catalysis and extraction using supercritical solutions (CESS), or biphasic systems wherein the catalysts are immobilized in an ionic liquid with the substrates and products being dissolved in $\mathrm{ScCO}_{2}$. The subject of enantioselective catalysis in $\mathrm{scCO}_{2}$ and the advantages of combined use of ionic liquids and $\mathrm{scCO}_{2}$ are discussed in Chapter 7.

\subsection{6}

\section{Phase-Transfer Catalysis}

For the above-discussed biphasic catalysis in liquid-liquid (e.g. aqueous-organic) systems, no additives are used, as the catalyst preferentially resides in a phase which is immiscible with that phase containing the product. One option to biphasic catalysis is to use amphiphilic additives such as phase-transfer catalysts which, by some interaction mechanism, can increase the mobility of a reactant or an intermediate into a favored phase, and thus provide an effective tool for accelerating the reaction and also controlling the selectivity. Phase-transfer catalysis (PTC) has been recognized for a long time, and typically involves simple experimental operations and mild reaction conditions, as well as environmentally friendly reagents and solvents [137]. The development of asymmetric PTC was triggered by a pioneering study performed by a Merck research group in 1984 [138]. Since then, several classes of chiral phase-transfer catalysts have been developed for use in asymmetric synthesis, mainly including cinchona alkaloid derivatives, purely synthesized chiral quaternary ammonium salts, and some chiral crown ethers. The synthetic utility of asymmetric PTC has been demonstrated for a wide range of reactions (most frequently involving anionic intermediates), among which the enantioselective alkylation of glycine derivatives has been most extensively studied and has become a powerful approach for the preparation of various $\alpha$-amino acids [139-146]. The reactions can be performed in either solid-organic or aqueousorganic biphasic systems, typically with moderate to relatively high catalyst loading (1 to $20 \mathrm{~mol} \%$ ). Although, in most cases it is difficult to recover the catalyst, recent efforts using polymer-supported cinchona alkaloid-derived ammonium salts or fluorous chiral phase-transfer catalyst have resulted in notable achievements towards this direction [146]. Several recent reviews summarizing the achievements and highlighting the future perspectives of asymmetric PTC are described in Chapter 11.

\subsection{7}

\section{Immobilization of Chiral Organic Catalysts}

Since the 1990s, organocatalysis has become established as an extremely important alternative methodology to enzymatic or organometallic catalysis in the field of asymmetric catalysis [147-150]. In general, an organic catalyst can be defined 
as a relatively simple low-molecular-weight organic compound which can promote a chemical transformation in substoichiometric quantity. By virtue of its metal-free nature an organocatalyst can, in principle, be advantageous in comparison with its metal-based counterpart in terms of reaction handling, catalyst stability and complete freedom of metal contamination in the product. Compared with naturally occurring catalysts (enzymes), organic catalysts are structurally simpler, more stable, less expensive, have a broader application scope and can demonstrate excellent selectivities in some cases. With regards to the catalyst immobilization, organic catalysts may also possess certain advantages over both enzymes (difficult to manipulate) and metal-based catalysts (generally suffer from metal leaching). Several types of achiral or chiral organic catalyst supported on either organic polymers (soluble or insoluble) or inorganic matrices have been developed for a range of reactions, and with varying degree of successes. Several reviews are available relevant to this subject [151-153], and these are detailed in Chapter 8. Although the development of immobilized chiral organic catalysts remains firmly in its infancy stage, the field of research is expected to expand greatly in the future in view of the present 'gold rush' in organocatalysis.

\section{3}

\section{Chirally Modified Metal Surface for Heterogeneous Asymmetric Hydrogenation}

Conceptually different from the above-discussed strategies that have been applied in the immobilization of homogeneous chiral catalysts, the modification of an active metal surface by an adsorbed chiral modifier has been shown to be very successful for heterogeneous asymmetric catalysis [154-166]. In this approach, an achiral solid (most frequently a metal surface) is used in combination with a suitable chiral organic compound (chiral modifier) as an inherently heterogeneous asymmetric catalysts. While the metal is responsible for catalytic activity, the surface-adsorbed chiral modifier can induce stereochemical control of the reaction via some type of mutual interaction with the substrate. Due to the many technical advantages associated with heterogeneous catalysis, such as easy separation and possible reuse of the catalyst and facile modification to continuous process operation, research in this field has been attracting a longstanding interest from both academia and industry. During its rather long history of development, this strategy has achieved prominent successes predominantly in the enantioselective hydrogenation of several types of prochiral substrate. A high substrate specificity is observed when using a chirally modified metal surface as a heterogeneous catalyst in hydrogenations, as by far only a limited number of metal-modifier-substrate combinations can provide enantioselectivities that are useful for synthetic applications. Three types of widely recognized efficient catalyst system have been developed based on the chiral modification of metal catalysts: (i) the nickel-tartrate- $\mathrm{NaBr}$ system for the hydrogenation of $\beta$-ketoesters, $\beta$-diketones and methyl ketones; (ii) the platinum-cinchona alkaloid system for the hydrogenation of $\alpha$-ketoesters, $\alpha$-ketoacids and lactones; and (iii) palladium catalysts modified with cinchona 
alkaloids for selected activated alkenes. Even though the substrate scope is still relatively narrow, high enantioselectivities which are useful for technical applications have been achieved in the hydrogenation of some specific reactants. The mechanism involved has also received much attention, the aim being to understand the adsorption of the modifier onto the metal surface and its interaction with the reactant, to interpret and predict the catalytic behaviors of the systems, as well as to facilitate the rational design of a suitable modifier. By using a combination of surface techniques, spectroscopic studies, computational modeling and kinetic studies, considerable mechanistic insight has been obtained, especially in the enantioselective pyruvate hydrogenation over Pt [162]. Nevertheless, a rational design of suitable metal/modifier catalytic systems still represents an extremely challenging goal at the present stage, and continuous endeavor in this direction should be of prime importance from both practical and theoretical viewpoints. Last, but not least, the use of an achiral heterogeneous catalyst and a chiral modifier as asymmetric heterogeneous catalyst is not limited to hydrogenations. For example, recently developed new catalyst-modifier systems based on NAP-MgO (nanocrystalline aerogel-prepared magnesium oxide) have proven very successful for a variety of asymmetric reactions $[167,168]$. Chirally modified metal nanoparticles have also been successfully tested in the enantioselective hydrosilylation of styrene [169], allylic alkylation [170], and Pauson-Khand-type reactions [171]. Although the catalyst-modifier systems other than those for hydrogenations are not described in Chapter 10 of this Handbook, these encouraging discoveries nonetheless underline the enormous application potential of the present strategy for asymmetric heterogeneous catalysis.

\section{4}

\section{Heterogeneous Enantioselective Catalysts in Industrial Research and Application}

As evidenced by the numerous publications in the field of heterogeneous asymmetric catalysis, over the years a large number of variously immobilized chiral catalysts - as well as several types of chirally modified catalyst-have been developed for a broad range of enantioselective reactions. Although some of these catalysts have demonstrated excellent performances in terms of activity, enantioselectivity, stability and/or reusability, the number of recognized industrial processes that use heterogeneous enantioselective catalysts for the commercial production of chiral compounds remains extremely small [172, 173]. As summarized in Chapter 12, to date only a few chirally modified heterogeneous hydrogenation catalysts (cinchona/Pt and $\mathrm{Ni} /$ tartaric acid/NaBr) are used industrially in the production of chiral intermediates. None of the immobilized chiral metal complexes has been applied industrially for large-scale production, despite several types of immobilized catalysts having demonstrated good potential for technical applications. Clearly, with few exceptions, most of the heterogeneous catalytic asymmetric methodologies developed to date have not been sufficiently mature to compete with alternative industrial methods (e.g. homogeneous catalysis, racemic 
mixture resolution). But this is not so surprising as it first appears, given the following general considerations. First, the inherent complexity associated with most heterogeneous systems makes the prediction of their catalytic performances extremely demanding. The often cumbersome immobilization procedure may considerably increase development time/costs, rendering the catalyst screening for a targeted reaction an even more challenging task. To make things worse, most of the immobilized metal complexes have suffered from problems of reduced activity and/or degraded enantioselectivity compared to their homogeneous counterparts as a result of the unintentional impact of supports on the active sites.

Nevertheless, we believe that heterogeneous asymmetric catalysis is-and will continue to be-an important field in the future, in view of its undisputable advantages over homogeneous counterparts with regards to separation and economy. To date, chemists have developed, with varying degrees of success, a wide variety of heterogenization techniques which will steadily broaden their applicability in the future. Finally, the identification of more economic and reliable heterogeneous asymmetric catalytic systems with high activity, selectivity, stability and reusability remains a challenging, but very worthwhile, goal which calls for collaborative efforts from both industry and academia.

\section{References}

1 Collins, A.N., Sheldrake, G.N. and Crosby, J. (1992) Chirality in Industry: The Commercial Manufacture and Applications of Optically Active Compounds, John Wiley \& Sons, Ltd, Chichester.

2 Collins, A.N., Sheldrake, G.N. and Crosby, J. (1997) Chirality in Industry II: Developments in the Commercial Manufacture and Applications of Optically Active Compounds, John Wiley \& Sons, Ltd, Chichester.

3 Sheldon, R.A. (1993) Chirotechnology: Industrial Synthesis of Optically Active Compounds, Dekker, New York.

4 Noyori, R. (1994) Asymmetric Catalysis in Organic synthesis, Wiley-Interscience, New York.

5 Ojima, I. (2000) Catalytic Asymmetric Synthesis, 2nd edn, Wiley-VCH Verlag $\mathrm{GmbH}$, New York.

6 Doyle, M. (1995) Advances in Catalytic Processes: Asymmetric Chemical Transformations, Vol. 1, JAI, Greenwich.

7 Jacobsen, E.N., Pfaltz, A. and Yamamoto, H. (1999) Comprehensive
Asymmetric Catalysis, Vol. I-III, Springer, Berlin.

8 Yamamoto, H. (2001) Lewis Acids in Organic Synthesis, Wiley-VCH Verlag $\mathrm{GmbH}$, New York.

9 Brunner, H. and Zettlmeier, W. (1993) Handbook of Enantioselective Catalysis. Vol. 1-2, Wiley-VCH Verlag GmbH, New York.

10 De Vos, D.E., Van-kelecom, I.F.J. and Jacobs, P.A. (2000) Chiral Catalyst Immobilization and Recycling, Wiley-VCH Verlag GmbH, Weinheim.

11 For a thematic issue on recoverable catalysts and reagents, see: Gladzsz, J.A., (2002) Chemical Reviews, 102, 3215-892.

12 Heitbaum, M., Glorius, F. and Escher, I. (2006) Angewandte Chemie-International Edition, 45, 4732-62.

13 Fan, Q.H., Li, Y.M. and Chan, A.S.C. (2002) Chemical Reviews, 102, 3385-465.

14 McMorn, P. and Hutchings, G.J. (2004) Chemical Society Reviews, 33, 108-22.

15 Baleizao, C. and Garcia, H. (2006) Chemical Reviews, 106, 3987-4043.

16 Corma, A. and Garcia, H. (2003) Chemical Reviews, 103, 4307-65. 
17 Hutchings, G.J. (1999) Chemical Communications, 301-6.

18 Fan, Q.H., Wang, R. and Chan, A.S.C. (2002) Bioorganic and Medicinal Chemistry Letters, 12, 1867-71.

19 Song, C.E. (2005) Annual Reports on the Progress of Chemistry Section C Physical Chemistry, 101, 143-73.

20 Jas, G. and Kirschning, A. (2003) Chemistry-A European Journal, 9, 5708-23.

21 Hodge, P. (2003) Current Opinion in Chemical Biology, 7, 362-73.

22 Kirschning, A., Solodenko, W. and Mennecke, K. (2006) Chemistry-A European Journal, 12, 5972-90.

23 Hodge, P. (2005) Industrial and Engineering Chemistry Research, 44, 8542-53.

24 Vankelecom, I.F.J. and Jacobs, P.A. (2000) Catalyst immobilization on inorganic supports, in Chiral Catalyst Immobilization and Recycling (eds D.E. De Vos, I.F.J. Van-kelecom and P.A. Jacobs), Wiley-VCH Verlag $\mathrm{GmbH}$, Weinheim, pp. 19-42.

25 Song, C.E. and Lee, S.G. (2002) Chemical Reviews, 102, 3495-524.

26 Corma, A. and Garcia, H. (2006) Advanced Synthesis Catalysis, 348, 1391-412.

27 De Vos, D.E., Dams, M., Sels, B.F. and Jacobs, P.A. (2002) Chemical Reviews, 102, 3615-40.

28 Li, C. (2004) Catalysis Reviews-Science and Engineering, 46, 419-92.

29 Song, C.E., Kim, D.H. and Choi, D.S. (2006) European Journal of Inorganic Chemistry, 2927-35.

30 Li, C., Zhang, H., Jiang, D. and Yang, Q. (2007) Chemical Communications, 547-58.

31 Bergbreiter, D.E. (2000) Organic polymers as a catalyst recovery vehicle, in Chiral Catalyst Immobilization and Recycling (eds D.E. De Vos, I.F.J. Vankelecom and P.A. Jacobs), Wiley-VCH Verlag GmbH, Weinheim, pp. 43-80.

32 Shuttleworth, S.J., Allin, S.M. and Sharma, P.K. (1997) Synthesis, 1217-39.

$33 \mathrm{Pu}, \mathrm{L}$. (1998) Tetrahedron: Asymmetry, 9 , 1457-77.

34 Saluzzo, C., Touchard, R., ter Halle, F., Fache, F., Schulz, E. and Lemaire, M.
(2000) Journal of Organometallic

Chemistry, 603, 30-9.

35 Clapham, B., Reger, T.S. and Janda, K.D. (2001) Tetrahedron, 57, 4637-62.

36 Bergbreiter, D.E. (2001) Current Opinion in Drug Discovery and Development, 4, 736-44.

37 Leadbeater, N.E. and Marco, M. (2002) Chemical Reviews, 102, 3217-73.

38 McNamara, C.A., Dixon, M.J. and Bradley, M. (2002) Chemical Reviews, 102, 3275-99.

39 Bräse, S., Lauterwasser, F. and Ziegert, R.E. (2003) Advanced Synthesis Catalysis 345, 869-929.

40 Haag, R. and Roller, S. (2004) Topics in Current Chemistry, 242, 1-42.

41 Mastrorilli, P. and Nobile, C.F. (2004) Coordination Chemical Reviews, 248, 377-95.

42 El-Shehawy, A.A. and Itsuno, S. (2005) Current Topics in Polymer Research, 1-69.

43 Dioos, B.M.L., Vankelecom, I.F.J. and Jacobs, P.A. (2006) Advanced Synthesis Catalysis, 348, 1413-46.

44 Gravert, D.J. and Janda, K.D. (1997) Chemical Reviews, 97, 489-509.

45 Bergbreiter, D.E. (1998) Catalysis Today, 42, 389-97.

46 Wentworth, P. Jr and Janda, K.D. (1999) Chemical Communications, 1917-24.

47 Toy, P.H. and Janda, K.D. (2000) Accounts of Chemical Research, 33, 546-54.

48 Bergbreiter, D.E. (2002) Chemical Reviews, 102, 3345-83.

49 Dickerson, T.J., Reed, N.N. and Janda, K.D. (2002) Chemical Reviews, 102, 3325-43.

50 Bergbreiter, D.E. and Li, J. (2004) Topics in Current Chemistry, 242, 113-76.

51 van de Coevering, R., Klein Gebbink, R.J.M. and van Koten, G. (2005) Progress in Polymer Science, 30, 474-90.

52 Bergbreiter, D.E. and Sung, S.D. (2006) Advanced Synthesis Catalysis, 348, 1352-66.

$53 \mathrm{Pu}$, L. (1998) Chemical Reviews, 98, 2405-94.

$54 \mathrm{Pu}, \mathrm{L}$. (1999) Chemistry - A European Journal, 5, 2227-32.

55 Astruc, D. and Chardac, F. (2001) Chemical Reviews, 101, 2991-3023. 
56 Kreiter, R., Kleij, A.W., Gebbink, R.J.M.K. and van Koten, G. (2001) Topics in Current Chemistry, 217, 163-99.

57 Crooks, R.M., Zhao, M., Sun, L., Chechik, V. and Yeung, L.K. (2001) Accounts of Chemical Research, 34, 181-90.

58 Oosterom, G.E., Reek, J.N.H., Kamer, P.C.J. and Van Leeuwen, P.W.N.M. (2001) Angewandte Chemie-International Edition, 40, 1828-49.

59 Twyman, L.J., King, A.S.H. and Martin, I.K. (2002) Chemical Society Reviews, 31, 69-82.

60 Van Heerbeek, R., Kamer, P.C.J., Van Leeuwen, P.W.N.M. and Reek, J.N.H. (2002) Chemical Reviews, 102, 3717-56.

61 King, A.S.H. and Twyman, L.J. (2002) Journal of the Chemical Society-Perkin Transactions 1, 2209-18.

62 Berger, A., Gebbink, R.J.M.K. and van Koten, G. (2006) Topics in Organometallic Chemistry, 20, 1-38.

63 Ribaudo, F., van Leeuwen, P.W.N.M. and Reek, J.N.H. (2006) Topics in Organometallic Chemistry, 20, 39-59.

64 Mery, D. and Astruc, D. (2006) Coordination Chemistry Reviews, 250, 1965-79.

65 Helms, B. and Frechet, J.M.J. (2006) Advanced Synthesis Catalysis, 348, 1125-48.

66 Brunner, H. and Altmann, S. (1994) Chemische Berichte, 127, 2285-96.

67 Brunner, H. (1995) Journal of Organometallic Chemistry, 500, 39-46.

68 Kassube, J.K. and Gade, L.H. (2006) Topics in Organometallic Chemistry, 20, 61-96.

69 Dai, L.X. (2004) Angewandte ChemieInternational Edition, 43, 5726-9.

70 Ding, K.-L., Wang, Z., Wang, X.-W., Liang, Y.-X. and Wang, X.-S. (2006) Chemistry-A European Journal, 12, 5188-97.

71 Kesanli, B. and Lin, W. (2003) Coordination Chemistry Reviews, 246, 305-26.

72 Ngo, H.L. and Lin, W. (2005) Topics in Catalysis, 34, 85-92.

73 Lin, W. (2005) Journal of Solid State Chemistry, 178, 2486-90.

\section{References $\mid 2$}

74 Williams, K.A., Boydston, A.J. and Bielawski, C.W. (2007) Chemical Society Reviews, 36, 729-44.

75 Cornils, B. (1995) Angewandte ChemieInternational Edition, 34, 1575-7.

76 Hanson, B.E. (2000) Liquid biphasic enantioselective catalysis, in Chiral Catalyst Immobilization and Recycling (eds D.E. De Vos, I.F.J. Van-kelecom and P.A. Jacobs), Wiley-VCH Verlag $\mathrm{GmbH}$, Weinheim, pp. 81-96.

77 Tzschucke, C.C., Markert, C., Bannwarth, W., Roller, S., Hebel, A. and Haag, R. (2002) Angewandte Chemie-International Edition, 41, 3964-4000.

78 Cole-Hamilton, D.J. (2003) Science, 299, 1702-6.

79 Keim, W. (2003) Green Chemistry, 5, 105-11.

80 Sheldon, R.A. (2005) Green Chemistry, 7, 267-78.

81 Baker, R.T., Kobayashi, S. and Leitner, W. (2006) Advanced Synthesis Catalysis, Special Issue $348(12+13)$ : Multiphase Catalysis, Green Solvents and Immobilization, 1317-771.

82 Liu, S. and Xiao, J. (2007) Journal of Molecular Catalysis A-Chemical, 270, 1-43.

83 Afonso, C.A.M., Branco, L.C., Candeias, N.R., Gois, P.M.P., Lourenco, N.M.T., Mateus, N.M.M. and Rosa, N. (2007) Chemical Communications, 2669-79.

$84 \mathrm{Li}$, C.J. and Chan, T.-H. (2007) Comprehensive Organic Reactions in Aqueous Media, 2nd edn, John Wiley \& Sons, Ltd, Hoboken.

85 Li, C.J. (1993) Chemical Reviews, 2023-35.

86 Lindstroem, U.M. (2002) Chemical Reviews, 102, 2751-71.

87 Li, C.J. (2005) Chemical Reviews, 105, 3095-165.

88 Li, C.J. and Chen, L. (2006) Chemical Society Reviews, 35, 68-82.

89 Hailes, H.C. (2007) Organic Process Research \& Development, 11, 114-20.

90 Cornils, B., Herrmann, W.A. and Eckl, R.W. (1997) Journal of Molecular Catalysis A-Chemical, 116, 27-33.

91 Cornils, B. and Herrmann, W.A. (2002) Immobilization by aqueous catalysts, in Applied Homogeneous Catalysis with Organometallic Compounds, 2nd edn, 
Wiley-VCH, Verlag GmbH, Weinheim, pp. 603-33.

92 Okuhara, T. (2002) Chemical Reviews, 102, 3641-65.

93 Vancheesan, S. and Jesudurai, D. (2002) Catalysis, 311-37.

94 Cornils, B. (1996) Applied Homogeneous Catalysis with Organometallic Compounds, Vol. 1 (eds B. Cornils and W. A. Herrmann), Wiley-VCH Verlag GmbH, Weinheim, Germany, pp. 577-600.

95 Kobayashi, S. and Manabe, K. (2002) Accounts of Chemical Research, 35, 209-17.

96 Sinou, D. (2002) Advanced Synthesis Catalysis, 344, 221-37.

97 Uozumi, Y. (2004) Topics in Current Chemistry, 242, 77-112.

98 Uozumi, Y. (2005) Catalysis Surveys from Asia, 9, 269-78.

99 Kobayashi, S. (2007) Pure and Applied Chemistry, 79, 235-45.

100 Horváth, I.T. and Rábai, J. (1994) Science, 266, 72-5.

101 Cornils, B. (1997) Angewandte ChemieInternational Edition, 36, 2057-9.

102 Horvath, I.T. (1998) Accounts of Chemical Research, 31, 641-50.

103 Fish, R.H. (1999) Chemistry-A European Journal, 5, 1677-80.

104 de Wolf, E., van Koten, G. and Deelman, B.J. (1999) Chemical Society Reviews, 28, 37-41.

105 Cavazzini, M., Montanari, F., Pozzi, G. and Quici, S. (1999) Journal of Fluorine Chemistry, 94, 183-93.

106 Barthel-Rosa, L.P. and Gladysz, J.A. (1999) Coordination Chemistry Reviews, 190-2, 587-605.

107 Horvath, I.T. (2002) Immobilization by other liquids: fluorous phases, in Applied Homogeneous Catalysis with Organometallic Compounds, 2nd edn, Vol. 2, Wiley-VCH Verlag GmbH, Weinheim, pp. 634-9.

108 Dobbs, A.P. and Kimberley, M.R. (2002) Journal of Fluorine Chemistry, 118, 3-17.

109 Gladysz, J.A. and Curran, D.P. (2002) Tetrahedron, 58, 3823-5.

110 Pozzi, G. and Shepperson, I. (2003) Coordination Chemistry Reviews, 242. 115-24.

111 Welton, T. (1999) Chemical Reviews, 99, 2071-83.
112 Wasserscheid, P. and Keim, W. (2000) Angewandte Chemie-International Edition, 39, 3772-89.

113 Sheldon, R. (2001) Chemical Communications, 2399-407.

114 Olivier-Bourbigou, H. and Magna, L. (2002) Journal of Molecular Catalysis A-Chemical, 182-183, 419-37.

115 Zhao, D., Wu, M., Kou, Y. and Min, E. (2002) Catalysis Today, 74, 157-89.

116 Dupont, J., de Souza, R.F. and Suarez, P.A.Z. (2002) Chemical Reviews, 102, 3667-91.

117 Zhao, H. and Malhotra, S.V. (2002) Aldrichimica Acta, 35, 75-83.

118 Gordon, C.M. (2001) Applied Catalysis A: General, 222, 101-17.

119 Baudequin, C. et al. (2003) Tetrahedron: Asymmetry, 14, 3081-93.

120 Welton, T. (2004) Coordination Chemistry Reviews, 248, 2459-77.

121 Muzart, J. (2006) Advanced Synthesis Catalysis, 348, 275-95.

122 Miao, W. and Chan, T.H. (2006) Accounts of Chemical Research, 39, 897-908.

123 Parvulescu, V.I. and Hardacre, C. (2007) Chemical Reviews, 107, 2615-65.

124 Parshall, G.W. (1972) Journal of the American Chemical Society, 94, 8716-19.

125 Chauvin, Y., Mussmann, L. and Olivier, H. (1995) Angewandte ChemieInternational Edition, 34, 2698-700.

126 Song, C.E. (2004) Chemical Communications, 1033-43.

127 Noyori, R. (1999) Supercritical fluids. Chemical Reviews Thematic Issue, 99, 353-634.

128 Baiker, A. (1999) Chemical Reviews, 99, 453-73.

129 Jessop, P.G., Ikariya, T. and Noyori, R. (1999) Chemical Reviews, 99, 475-93.

130 Oakes, R.S., Clifford, A.A. and Rayner, C.M. (2001) Journal of the Chemical Society-Perkin Transactions 1, 917-41.

131 Musie, G., Wei, M., Subramaniam, B. and Busch, D.H. (2001) Coordination Chemistry Reviews, 219-221, 789-820.

132 Leitner, W. (2002) Accounts of Chemical Research, 35, 746-56.

133 Licence, P., Ke, J., Sokolova, M., Ross, S.K. and Poliakoff, M. (2003) Green Chemistry, 5, 99-104.

134 Campestrini, S. and Tonellato, U. (2005) Current Organic Chemistry, 9, 31-47. 
135 Burk, M.J., Feng, S.G., Gross, M.F. and Tumas, W. (1995) Journal of the American Chemical Society, 117, 8277-8.

136 Cole-Hamilton, D.J. (2006) Advanced Synthesis Catalysis, 348, 1341-51.

137 Makosza, M. (2000) Pure and Applied Chemistry, 72, 1399-403.

138 Dolling, U.-H., Davis, P. and Grabowski, E.J.J. (1984) Journal of the American Chemical Society, 106, 446-7.

139 Nelson, A. (1999) Angewandte ChemieInternational Edition, 38, 1583-5.

140 O’Donnell, M.J. (2001) Aldrichimica Acta, 34, 3-15.

141 Maruoka, K. and Ooi, T. (2003) Chemical Reviews, 103, 3013-28.

142 O'Donnell, M.J. (2004) Accounts of Chemical Research, 37, 506-17.

143 Lygo, B. and Andrews, B.I. (2004) Accounts of Chemical Research, 37, 518-25.

144 Vachon, J. and Lacour, J. (2006) Chimia, 60, 266-75.

145 Lygo, B. and Beaumont, D.J. (2007) Chimia, 61, 257-62.

146 Ooi, T. and Maruoka, K. (2007) Angewandte Chemie-International Edition, 46, 4222-66.

147 List, B. (2005) Organic and Biomolecular Chemistry, 3, 719.

148 Dalko, P.I. and Moisan, L. (2001) Angewandte Chemie-International Edition, 40, 3726-48.

149 Dalko, P.I. and Moisan, L. (2004) Angewandte Chemie-International Edition, 43, 5138-75.

150 Berkessel, A. and Gröger, H. (2004) Asymmetric Organocatalysis, Wiley-VCH Verlag GmbH, Weinheim.

151 Benaglia, M., Puglisi, A. and Cozzi, F. (2003) Chemical Reviews, 103, 3401-29.

152 Cozzi, F. (2006) Advanced Synthesis Catalysis, 348, 1367-90.

153 Benaglia, M. (2006) New Journal of Chemistry, 30, 1525-33.

154 Blaser, H.-U., Jalett, H.P., Müller, M. and Studer, M. (1997) Catalysis Today, 37, 441-63.

155 Wells, P. B. and Wilkinson, A.G. (1998) Topics in Catalysis, 5, 39-50.

156 Studer, M., Blaser, H.-U. and Exner, C. (2003) Advanced Synthesis Catalysis, 345, 45-65.

157 Wells, P. B. and Wells, R.P.K. (2000) Enantioselective hydrogenation
References |23

catalyzed by platinum group metals modified by natural alkaloids, in Chiral Catalyst Immobilization and Recycling (eds D.E. De Vos, I.F.J. Vankelecom and P.A. Jacobs), Wiley-VCH Verlag GmbH, Weinheim, pp. 123-54.

158 Baiker, A. (2000) Design of new chiral modifiers for heterogeneous enantioselective hydrogenation: a combined experimental and theoretical approach, in Chiral Catalyst Immobilization and Recycling (eds D.E. De Vos, I.F.J. Vankelecom and P.A. Jacobs), Wiley-VCH Verlag GmbH, Weinheim, pp. 155-71.

159 Tai, A. and Sugimura, T. (2000) Modified Ni catalysts for enantio-differentiating hydrogenation, in Chiral Catalyst Immobilization and Recycling (eds D.E. De Vos, I.F.J. Vankelecom and P.A. Jacobs), Wiley-VCH Verlag GmbH, Weinheim, pp. 173-209.

160 Osawa, T., Harada, T. and Takayasu, O. (2000) Topics in Catalysis, 13, 155-68.

161 von Arx, M., Mallat, T. and Baiker, A. (2002) Topics in Catalysis, 19, 75-87.

162 Buergi, T. and Baiker, A. (2004) Accounts of Chemical Research, 37, 909-17.

163 Osawa, T., Harada, T. and Takayasu, O. (2006) Current Organic Chemistry, 10 , 1513-31.

164 Bartok, M. (2006) Current Organic Chemistry, 10, 1533-67.

165 Studer, M. and Blaser, H.U. (2006) Enantioselective hydrogenation of activated ketones using heterogeneous Pt catalysts modified with cinchona alkaloids, in Handbook of Chiral Chemicals, 2nd edn, CRC Press LLC, Boca Raton, pp. 345-57.

166 Mallat, T., Orglmeister, E. and Baiker, A. (2007) Chemical Reviews, 107, 4863-90.

167 Choudary, B.M., Kantam, M.L., Ranganath, K.V.S., Mahendar, K. and Sreedhar, B. (2004) Journal of the American Chemical Society, 126, 3396-7.

168 Choudary, B.M., Ranganath, K.V.S., Pal, U., Kantam, M.L. and Sreedhar, B. (2005) Journal of the American Chemical Society, 127, 13167-71.

169 Tamura, M. and Fujihara, H. (2003) Journal of the American Chemical Society, 125, 15742-3.

170 Jansat, S., Gómez, M., Philippot, K., Muller, G., Guiu, E., Claver, C., Castillón, 
S. and Chaudret, B. (2004) Journal of the American Chemical Society, 126, 1592-3.

171 Park, K.H. and Chung, Y.K. (2005) Advanced Synthesis Catalysis, 347, 854-66.

172 Blaser, H.U., Pugin, B. and Studer, M. (2000) Enantioselective heterogeneous catalysis: academic and industrial challenges, in Chiral Catalyst Immobilization and Recycling (eds D.E. De Vos, I.F.J. Vankelecom and P.A. Jacobs), Wiley-VCH Verlag GmbH, Weinheim, pp. 1-17.

173 End, N. and Schoening, K.U. (2004) Topics in Current Chemistry, 242, 241-71. 